\title{
VIOLÊNCIA DOMÉSTICA E STALKING PÓS-RUTURA: DINÂMICAS, COPING E IMPACTO PSICOSSOCIAL NA VÍTIMA
}

Célia Ferreira ${ }^{1}$

Marlene Matos $^{2}$

[Enviado a 29-05-2012. Aceite a 20-09-2012]

Resumo: Este artigo reflete uma realidade pouco conhecida no plano nacional - o stalking após a rutura de uma relação abusiva. Nesse contexto, o objectivo era conhecer as dinâmicas associadas a esta modalidade de vitimação, as respostas das vítimas face à mesma e os preditores de desajustamento psicossocial na vítima. $\mathrm{A}$ amostra foi constituída por 104 mulheres que foram vítimas de abuso na relação e de stalking após o seu término. Os resultados ilustram a natureza prolongada do stalking e documentam um continuum de ações que engloba atos com uma gravidade bastante distinta. A maioria das inquiridas relatou medo face ao stalking e admitiu que este influiu negativamente na sua vida. Apesar de tudo, as vítimas revelaram-se ativas na gestão deste tipo de violência, empreendendo diferentes estratégias de coping. A frequência média dos comportamentos de stalking e o coping de não evitamento surgiram como preditores de desajustamento psicossocial. É, pois, necessário um maior investimento ao nível da investigação e das práticas para combater este problema.

Palavras-Chave: Stalking, Violência doméstica, Rutura, Reações emocionais, Coping e Impacto psicossocial

Domestic violence and post-relationship stalking: Dynamics, coping and victim's psycho-social impact (Abstract): This article reflects on a nationally insufficiently known reality - stalking after the breakup of an abusive relationship. Being so, the

${ }^{1}$ Escola de Psicologia, Universidade do Minho, Campus de Gualtar, 4710-057 Braga. ISMAI - Instituto Superior da Maia, Av. Carlos Oliveira Campos - Castelo da Maia, 4475-690 Avioso, S. Pedro

2 Escola de Psicologia, Universidade do Minho, Campus de Gualtar, 4710-057 Braga. Endereço eletrónico para correspondência: Célia Ferreira; celia.psi@gmail.com 
aim was to get to know the dynamics associated to this type of victimisation, the answers victims gave to it and the predictors of psycho-social maladjustment on the victim. The sample was constituted by 104 women who were victims of abuse during the ex-relationship and of stalking after they ended the relationship. Results have pointed out the prolonged nature of stalking and documented a continuum of actions referring to extremely different acts on what concerns their seriousness. The majority of the victims reported fear when dealing with stalking and admitted it had a negative influence on their lives. However, these women have assumed an active position when dealing with it and have developed different coping strategies. The mean frequency of stalking behaviours and unavoidable coping came out as predictors of psycho-social maladjustment. So, a greater investment on what concerns research and practices to fight this problem is needed.

Keywords: Stalking, Domestic violence, Breakup, Emotional Reactions, Coping and Psycho-social impact

O stalking tem sido entendido como um "fenómeno social e comportamental complexo" (O’Connor \& Rosenfeld, 2004, p. 4), sendo possível encontrar na literatura diferentes definições para o problema (Spitzberg, 2002; Spitzberg \& Cupach, 2007). Porque nenhuma definição linear nos parece capaz de captar a pluralidade de experiências envolvidas nesta realidade, destacamos aquela que, pela sua amplitude, parece mais integradora: "padrão de comportamentos de assédio persistente, que se traduz em formas diversas de comunicação, contacto, vigilância e monitorização de uma pessoa" (Grangeia \& Matos, 2010, p. 124), o qual assume um caráter intrusivo e/ou indesejado para o alvo e pode ser mediado por motivações diversas.

De facto, o stalking inclui um espectro diversificado de comportamentos, desde atos aparentemente "inócuos" (e.g., oferecer flores) até outros explicitamente intimidatórios (e.g., ameaças, perseguição), que tendem a escalar em frequência e gravidade ao longo do tempo, podendo mesmo associar-se a outras formas de violência física e/ou sexual (cf., Spitzberg \& Cupach, 2007).

Embora os comportamentos de stalking não constituam uma novidade (e.g., Meloy, 2007), aquilo que hoje se reconhece como uma forma particular de violência foi, durante séculos, socialmente aceite e até reforçado, com base nos ideais do romantismo e da paixão (Mullen, Pathé, \& Purcell, 2001). Numa análise da literatura internacional sobre o fenómeno percebe-se que o termo apenas adquiriu visibilidade social no início dos anos 90, quando os media norte-americanos começaram a usar esta designação para descrever situações de perseguição a figuras - públicas (Lowney \& Best, 1995). A atenção mediática dedicada a estes casos desencadeou o reconhecimento 
público do problema e patrocinou um conjunto de reformas legislativas com vista à criminalização da conduta (iniciadas na Califórnia em 1990). Mais tarde (entre 1992 e 1994), grupos feministas e de apoio às vítimas vieram defender um enquadramento mais lato para o fenómeno, importando o conceito para o domínio da violência doméstica (idem).

Os inquéritos de vitimação posteriormente conduzidos neste domínio em diferentes países (cf., Grangeia \& Matos, 2010) serviram para denunciar a elevada prevalência do problema e a sua transversalidade, fornecendo sustentação empírica à concetualização do stalking como um fenómeno de violência interpessoal, genderizado, frequentemente perpetrado sobre mulheres por homens no contexto de relações íntimas (cf., Spitzberg \& Cupach, 2007).

Neste contexto específico, embora os estudos mostrem que os comportamentos de stalking podem emergir em diferentes momentos da trajetória relacional, entendemos que a sua concetualização terá de ter em conta as motivações que lhe estão subjacentes. Tipicamente, dois propósitos fundamentais presidem a este padrão de conduta: a tentativa de reconciliação e/ou o desejo de vingança pela separação, sendo certo que muitos destes stalkers facilmente flutuam entre estes dois pólos (e.g., Burgess, Harner, Baker, Hartman, \& Lole, 2001; Morewitz, 2003). Neste sentido, cruzando estas duas dimensões, concetualizamos o momento pós-rutura como o mais vulnerável para a emergência desta modalidade de vitimação enquanto padrão específico de violência (embora reconheçamos que comportamentos desta natureza possam emergir ainda durante o curso da união).

O stalking neste contexto não passou despercebido junto dos investigadores, estabelecendo-se hoje como um campo fértil de estudo e reflexão. Atualmente, é consensual que o stalking constitui uma faceta específica e particular da violência na intimidade, embora esteja sobejamente documentada a sua relação com outras formas de abuso na esfera relacional (e.g., Basile \& Hall, 2010; Miller, 2006).

Comparativamente a outros cenários de stalking (e.g., entre desconhecidos, no contexto de relações profissionais), os dados empíricos ilustram que o stalking perpetrado por ex-parceiros é caracterizado por uma maior diversidade e frequência de estratégias (e.g., Mohandie, Meloy, McGowan, \& Williams, 2006) e um maior risco de violência física, de persistência e reincidência dos comportamentos (cf., McEwan, Mullen, \& Purcell, 2007). A literatura da especialidade tem mesmo identificado o stalking neste contexto como um importante fator de risco para a ocorrência de formas potencialmente letais de violência, reforçando a elevada perigosidade associada a este tipo de conduta (e.g., Campbell, Glass, Sharps, Laughon, \& Bloom, 2007).

Segundo Logan e Walker (2009), este tipo de stalkers pode ver a sua "missão" facilitada pelo facto de possuir informações pormenorizadas sobre as 
rotinas da vítima, conhecer bem nichos da sua vida privada (e.g., preocupações, fragilidades) e por usufruir, quase sempre, de mais oportunidades de comtacto com aquela, principalmente se tiverem filhos e/ou amigos em comum; além disso, a escalada para atos de violência mais severa poderá ser facilitada porque muitas barreiras foram já quebradas durante a relação passada.

Para além do risco e dos custos macro-económicos que decorrem da vitimação por stalking (Centers for Disease Control and Prevention, 2003 como citado em Spitzberg \& Cupach, 2007), os autores que estudam o fenómeno e os clínicos que nele intervêm são unânimes quanto aos custos individuais que esta conduta acarreta para as vítimas, designadamente ao nível do estilo de vida, ao nível da saúde física e, em particular, ao nível da saúde psicológica (cf. Mechanic, 2002).

Especificamente, a literatura sobre os efeitos do stalking no âmbito de relacionamentos íntimos ilustra várias consequências emocionais associadas a este tipo de experiência, traduzidas em condições de grande vulnerabilidade pessoal (Brewster, 1998; Kamphuis, Emmelkamp, \& Bartak, 2003; Logan, Shannon, Cole, \& Walker, 2006). Por exemplo, num estudo conduzido junto de uma amostra de 187 mulheres vítimas de stalking após a rutura relacional, Brewster (1998) concluiu que praticamente todas (99\%) experienciaram uma redução na qualidade de vida em consequência da conduta levada a cabo pelo ex-parceiro; além disso, as vítimas relataram uma grande diversidade de respostas emocionais, nomeadamente desconfiança $(44.4 \%)$, medo $(41.7 \%)$, nervosismo ou agitação $(31 \%)$, raiva $(26.7 \%)$, paranóia $(35.7 \%)$ e sintomatologia depressiva (21.4\%).

Também o trabalho de Mechanic, Uhlmansiek, Weaver e Resick (2002) documenta a extensão dos sintomas psicológicos numa amostra de mulheres batidas vítimas de stalking. Os dados ilustram que aquelas que foram alvo de stalking mais severo manifestavam índices superiores de indicadores traumáticos; além disso, apesar de todas as vítimas exibirem níveis elevados de depressão, os sintomas depressivos mais severos estavam associados a experiências de stalking, também, mais severas. Contudo, é necessário explorar mais detalhadamente o efeito individual do stalking nas vítimas.

Para além das respostas emocionais, a reação das vítimas ao stalking contempla outro tipo de respostas, de natureza estratégica (Dutton, 1993, 1996 como citado em Mechanic, Uhlmansiek et al., 2002), comummente designadas na literatura como estratégias de coping. Os comportamentos empreendidos pelas vítimas podem assumir múltiplas formas, podendo passar, por exemplo, pela alteração de contactos pessoais, pela implementação de medidas de segurança, pela mudança de residência ou pela procura de apoio formal e/ou informal (e.g., Brewster, 1998; Mechanic, Uhlmansiek et al., 2002). Spitzberg e Cupach (2001, 2007; Spitzberg, 2002) criaram uma tipologia de coping que organiza as estratégias tipicamente adotadas pelas 
vítimas de stalking (em geral) em cinco principais categorias: Moving With, Moving Against, Moving Away, Moving Inward e Moving Outward. As estratégias de Moving With representam esforços da vítima para negociar com o stalker, visando uma resolução pacífica do problema; contudo, porque podem ser racionalizadas por aquele, são consideradas ineficazes e potencialmente reforçadoras dos comportamentos de stalking. As estratégias de Moving Against constituem tentativas da vítima para confrontar o stalker (e.g., ameaçar, agredir) e são também desaconselhadas, não só porque patrocinam oportunidades de interação, mas também porque podem despoletar represálias e condicionar a credibilidade de ações futuras (e.g., ameaçar chamar a polícia). Por sua vez, as vítimas podem optar por comportamentos do tipo Moving Away, através dos quais procuram evitar o stalker e qualquer possibilidade de contacto ou interação com aquele. Apesar de estas estratégias terem a desvantagem de atribuir à vítima maior responsabilidade pelo curso do stalking, são tidas como as mais eficazes a curto e longo-prazo. As estratégias de Moving Inward ilustram a opção da vítima em negar, minimizar ou redefinir a situação, por exemplo, através do recurso à medicação ou consumo de substâncias; embora possam ser úteis na gestão a curto prazo do stress inerente à vitimação sofrida, é pouco provável que contribuam para alterar a conduta do stalker e podem mesmo deixar a vítima mais vulnerável a futuros ataques. Finalmente, as opções tipificadas como Moving outward centram-se na procura de apoio junto de terceiros, quer formal quer informal, e são globalmente tidas como eficazes.

Porém, os trabalhos que têm produzido evidências científicas sobre coping nas situações de stalking em contexto de relacionamentos íntimos encerram algumas limitações relevantes: baseiam as suas conclusões em exemplos de estratégias particulares - versus categorias de coping mais abrangentes (e.g., Brewster, 1998; Mechanic, Uhlmansiek et al., 2002) ou recorrem exclusivamente a amostras de estudantes universitários (e.g., Dutton \& Winstead, 2010).

Concomitantemente, importa não ignorar que aquilo que sabemos acerca do stalking no âmbito de relações íntimas continua a chegar-nos, quase exclusivamente, de investigações noutros países. Em Portugal, esta realidade tem sido pouco estudada, embora, tal como sublinhado por alguns autores (e.g., O'Connor \& Rosenfeld, 2004; Sheridan, Blaauw, \& Davies, 2003), a singularidade desta forma de vitimação imponha a necessidade de novos contributos, capazes de patrocinar um conhecimento mais profundo e contextualizado do fenómeno, sensível a realidades e influências socioculturais particulares.

Assim, na origem deste trabalho estiveram motivações diversas. Desde logo, a ausência de investigações nacionais acerca desta temática específica levanta muitas questões, de natureza variada. Por outro lado, procurou- 
-se através deste trabalho colmatar outras lacunas presentes na literatura internacional, nomeadamente analisar, através do recurso a categorias abrangentes, o tipo de respostas emocionais e estratégicas associadas a este cenário específico de violência, assim como discriminar o impacto específico das diferentes formas de violência sofridas pelas vítimas de violência durante a relação e de stalking após a rutura.

Assim, através da experiência de mulheres vítimas, pretendeu-se com este estudo quantitativo (1) caracterizar as dinâmicas do stalking pós-rutura, explorando a sua relação com características da ex-relação; (2) identificar as respostas emocionais consequentes a esta forma de vitimação e o tipo de estratégias de coping adotadas pelas vítimas; e (3) avaliar o impacto psicossocial exibido pelas vítimas, identificando os fatores preditores de desajustamento psicossocial clinicamente significativo.

\section{Método}

\section{Participantes}

A idade das participantes estava compreendida entre os 19 e os 69 anos, com uma média de 37.64 anos $(D P=11.09)$. De acordo com aquelas, a idade dos respetivos stalkers variava entre os 16 e os 70 anos, com uma média de 41.36 anos $(D P=11.41)$.

A maioria das vítimas $(85.4 \%)$ era de nacionalidade portuguesa. Do total, $35.9 \%$ residia na região norte do país e $42.7 \%$ na região centro. No que concerne às habilitações literárias, $3 / 4$ das participantes possuía um grau de qualificação igual ou inferior ao $3^{\circ}$ Ciclo do Ensino Básico. A maioria pertencia à população ativa (66\%), estando as restantes desempregadas, reformadas ou a estudar. Em média, as vítimas auferiam 532.73 euros mensais $(D P=365.58)$. Mais de metade da amostra $(64.4 \%)$ classificou o seu nível sócio-económico (NSE) como baixo ou médio-baixo.

À data da realização do estudo, $26.3 \%$ das participantes afirmou estar envolvida num novo relacionamento íntimo, embora a maioria $(69.2 \%)$ não coabitasse com o novo companheiro (cf. Tabela 1). 
Tabela 1: Características sociodemográficas da amostra

\begin{tabular}{|c|c|}
\hline & $M(D P ;$ Min., Max. $) / \%\left(N^{\mathrm{a}}\right)$ \\
\hline Idade da vítima & $37.64(11.09 ; 19,69)$ \\
\hline Idade do stalker & $41.36(11.41 ; 16,70)$ \\
\hline \multicolumn{2}{|l|}{ Nacionalidade } \\
\hline Portugal & $85.4(88)$ \\
\hline Países da América do Sul & $4.9(5)$ \\
\hline PALOP's & $3.9(4)$ \\
\hline Países do Leste Europeu & $1.9(2)$ \\
\hline Outros Países Europeus & $3.9(4)$ \\
\hline \multicolumn{2}{|l|}{ Região do País } \\
\hline Norte & $35.9(37)$ \\
\hline Centro & $42.7(44)$ \\
\hline Lisboa e Vale do Tejo & $11.7(12)$ \\
\hline Algarve & $1.9(2)$ \\
\hline R.A. Açores & $2.9(3)$ \\
\hline R.A. Madeira & $4.9(5)$ \\
\hline \multicolumn{2}{|l|}{ Habilitações Literárias } \\
\hline $1^{\circ}$ Ciclo EB & $23.8(24)$ \\
\hline $2^{\circ}$ Ciclo EB & $25.7(26)$ \\
\hline $3^{\circ}$ Ciclo EB & $25.7(26)$ \\
\hline Secundário & $12.9(13)$ \\
\hline Licenciatura & $11.9(12)$ \\
\hline \multicolumn{2}{|l|}{ Situação Laboral } \\
\hline Empregada & $66(68)$ \\
\hline Desempregada & $28.2(29)$ \\
\hline Reformada & $1.9(2)$ \\
\hline Estudante & $3.9(4)$ \\
\hline Rendimento Mensal (€) & $532.73(365.58 ; 0,2600)$ \\
\hline \multicolumn{2}{|l|}{ NSE } \\
\hline Baixo & $40.6(41)$ \\
\hline Médio-Baixo & $23.8(24)$ \\
\hline Médio & $29.7(30)$ \\
\hline Médio-Alto & $5.9(6)$ \\
\hline \multicolumn{2}{|l|}{ Envolvimento amoroso } \\
\hline Sem relação atual & $73.7(73)$ \\
\hline Com relação atual & $26.3(26)$ \\
\hline \multicolumn{2}{|l|}{ Coabitação } \\
\hline Não & $69.2(18)$ \\
\hline Sim & $30.8(8)$ \\
\hline
\end{tabular}

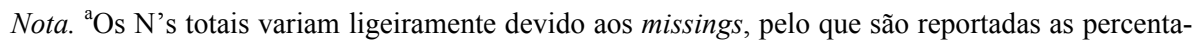
gens válidas. 


\section{Instrumentos}

- Inventário de Violência Conjugal - Parte B - Versão 3 (I.V.C.-B-3; C. Machado, M. Matos, \& M. Gonçalves, 2006, adaptado por C. Ferreira \& M. Matos, 2009): Este instrumento de autorrelato permite identificar a vitimação e perpetração de comportamentos abusivos durante as relações de intimidade. É composto por 21 itens (o último dos quais com a opção "Outros"), que correspondem a comportamentos emocional e fisicamente abusivos (e.g., "Gritar ou ameaçar, para meter medo"; "Dar uma sova"). Cada item é avaliado numa escala de frequência de 3 pontos ( 0 = "Nunca", 1 = "Uma vez" e 2 = "Mais do que uma vez"). Para este estudo foi utilizada apenas a Parte B do instrumento (relativa a relações passadas) e foi dada a instrução às participantes para que respondessem com base no relacionamento que mantiveram com o stalker, utilizando a designação "ex-parceiro".

- Inventário de Comportamentos de Stalking - Versão 2 (I.C.S.-2; H. Grangeia, M. Matos, \& C. Machado, 2008, adaptado por C. Ferreira \& M. Matos, 2009): Este instrumento de autorrelato permite identificar a vitimação por stalking, sendo composto por 36 itens (o último dos quais com a opção "Outros"), que correspondem a três tipos de comportamentos (Grangeia \& Matos, em preparação): 'Cortejamento \& Aproximação' (estratégias de comunicação e/ou contacto com o objetivo de expressar afeto ou outros sentimentos; e.g., "Deu, ou deixou para serem encontrados, presentes"), 'Assédio \& Invasão' (estratégias para obter informações sobre a vítima, invadir a sua privacidade ou individualidade; e.g., "Vigiou ou controlou o meu comportamento") e 'Ameaças \& Violência' (ações interpostas para influenciar o comportamento da vítima ou provocar dano real; e.g., "Agarrou-me ou impediu-me de continuar o meu percurso"). Cada item é avaliado numa escala de frequência de 5 pontos ( 0 = "Nunca", 1 = "Uma vez", 2 = "2 a 3 vezes", 3 = "4 a 5 vezes", 4 = "Mais de 5 vezes"). Para este estudo foi dada a instrução às participantes para que respondessem com base na conduta levada a cabo pelo ex-parceiro após o término do relacionamento.

- Outcome Questionnaire - 45.2 (OQ-45.2; Lambert \& Burlingame, 1996, versão portuguesa adaptada por Machado \& Fassnacht, em preparação): Este instrumento de autorrelato foi selecionado por fornecer uma medida do ajustamento e perturbação psicossocial dos indivíduos. É composto por 45 itens de tipo Likert, cotados numa escala de "Nunca" (0) a "Quase sempre" (4). Neste estudo, foi apenas considerado o score total. O Alpha de Cronnbach obtido nesta amostra de investigação $(\alpha=.92)$ sugere uma boa consistência interna.

- Questionário padronizado sobre: (i) Características sociodemográficas; (ii) Outras características da ex-relação, nomeadamente duração, tipo, sexo do ex-parceiro, perceção face à qualidade do relacionamento e tempo 
decorrido desde a rutura; (iii) Outras características do stalking pós-rutura, especificamente curso, duração, sentimentos face à experiência, forma como a mesma afetou as suas vidas e como as vítimas a avaliavam; (iv) Tipo de estratégias de coping adotadas face aos comportamentos de stalking sofridos. Especificamente, face a um conjunto de seis diferentes tipos de estratégias (adaptado de Spitzberg \& Cupach, 2001, 2007; Spitzberg, 2002), pediu-se às participantes que indicassem todas aquelas que empreenderam, pelo menos uma vez, como resposta aos comportamentos de stalking perpetrados pelo ex-parceiro (e.g., "Negociei, de forma pacífica, com o meu ex-parceiro de modo a que ele terminasse estes comportamentos"; "Procurei apoio junto de amigos ou familiares").

\section{Procedimentos para seleção da amostra e recolha dos dados}

Para este estudo foram contactadas diversas instituições de apoio a vítimas, solicitando a sua colaboração através da identificação de potenciais participantes que, aceitando colaborar nesta investigação, cumprissem os critérios de inclusão definidos, nomeadamente: (i) ser do sexo feminino e (ii) ter sido alvo, em algum momento das suas vidas, de assédio persistente por parte de um ex-parceiro íntimo do sexo masculino, traduzido em comportamentos de perseguição, vigilância, monitorização, intimidação, ameaça ou de outras formas de comunicação ou contacto, repetido e indesejado. Sublinhe-se que no contacto com as instituições foi sempre utilizado o conceito de 'assédio persistente' uma vez que o termo 'stalking' encontra-se ainda pouco difundido na sociedade portuguesa.

A recolha dos dados efetuou-se entre fevereiro de 2009 e janeiro de 2010. A administração ocorreu nas instituições de apoio que colaboraram, com um tempo médio de resposta de 30 minutos. Não foi dada às participantes qualquer compensação económica e aquelas foram sempre devidamente informadas acerca do caráter anónimo e voluntário da sua participação, tendo fornecido o seu consentimento informado para a participação nesta investigação.

No total, 106 vítimas de stalking pós-rutura completaram o questionário. Destas, $98.1 \%(n=104)$ foram alvo de maus tratos físicos e/ou emocionais durante a relação com o stalker. Atendendo a esta preponderância, apenas as vítimas com história de violência durante a ex-relação foram incluídas na análise, perfazendo uma amostra final de 104 mulheres vítimas.

\section{Resultados}

Para a realização de todas as análises estatísticas recorremos ao software informático Statistical Package for the Social Sciences (SPSS), versão 18.0. 
Em primeiro lugar, começámos por analisar os dados descritivos relativos às dinâmicas de violência, referentes aos maus tratos sofridos e perpetrados durante a ex-relação, assim como à conduta de stalking experienciada após a rutura. Investigámos de seguida a relação entre as características da ex-relação e as características do stalking sofrido após a separação.

Em segundo lugar, caracterizámos as respostas emocionais e as estratégias de coping face à campanha de stalking vivenciada. Analisámos ainda o nível de impacto psicossocial manifestado pelas participantes à data da realização do estudo, assim como a relação entre esta variável de sintomatologia e outras variáveis do estudo, nomeadamente características socio-demográficas, características da ex-relação, característica da campanha de stalking e tipo de estratégias de coping empreendidas.

A este propósito, importa sublinhar que sempre que trabalhámos com variáveis intervalares, começámos por conduzir uma análise exploratória de dados de forma a verificar se estavam cumpridos os pressupostos subjacentes à utilização de testes paramétricos. Tendo verificado que tais requisitos não estavam reunidos, utilizámos a estratégia de calcular ambos os testes: paramétricos (t-student e Coeficiente de Correlação de Pearson) e seus equivalentes não paramétricos (Mann-Whitney e Coeficiente de Correlação de Spearman, respectivamente). Tal como aconselhado por Fife-Schaw (2000), sempre que as conclusões retiradas dos dois conjuntos de testes se mantinham, optámos por apresentar os resultados dos testes paramétricos; nos casos em que as conclusões eram discrepantes, apresentámos os resultados dos testes não paramétricos.

Por último, procedemos a uma análise de regressão logística para identificar os fatores preditores de desajustamento clínico.

\section{Características e dinâmicas de vitimação}

Características da ex-relação íntima

Todas as relações passadas entre a vítima e o stalker eram de natureza heterossexual. Especificamente, mais de metade das vítimas $(55.8 \%, n=58)$ esteve casada com o respetivo stalker, tendo $41.3 \%(n=43)$ vivido em união de facto e $2.9 \%(n=3)$ mantido um relacionamento de namoro com aquele (sem coabitação). Em média, estes relacionamentos duraram 156.05 meses (aproximadamente 13 anos), variando entre 1 e 576 (i.e., 48 anos) $(D P=$ 126.75).

Durante a ex-relação, e tal como referido anteriormente, todas as participantes $(100 \%, n=104)$ foram alvo de violência, tendo sofrido, em média, mais de 13 atos abusivos distintos $(M=13.45, D P=5.1)$. Simultaneamente, $55.8 \%(n=58)$ das inquiridas reconheceu ter dirigido algum ato desta natu- 
reza contra o seu ex-parceiro: entre 1 e 10 atos distintos de violência perpetrados $(M=3.16, D P=2.33)$.

Considerando a frequência média dos diferentes tipos de abuso descritos no IVC-3, verificou-se que as vítimas sofreram violência emocional e violência física de forma reiterada $(M=1.44, D P=0.45 ; M=1.13, D P=$ 0.59 , respetivamente). Por sua vez, perpetraram qualquer uma destas formas de mau-trato contra o parceiro num registo ocasional ( $M$ ' Violência Emocional' perpetrada $=0.32, D P=0.28 ; M$ 'Violência física perpetrada' $=0.18$, $D P=0.19)$. Para efeito das restantes análises, considerámos apenas a violência sofrida pelas vítimas durante a relação passada com o stalker.

O relacionamento passado foi avaliado por $41.3 \%(n=43)$ das vítimas como "extremamente negativo e como "negativo" em $36.5 \%(n=38)$ dos casos. Das restantes vítimas, $17.3 \%(n=18)$ forneceu uma resposta ambígua ("nem positivo nem negativo"), 1.9\% $(n=2)$ considerou a ex-relação "positiva" e $2.9 \%(n=3)$ avaliou-a como "extremamente positiva".

Em média, a rutura do relacionamento com o stalker tinha ocorrido há 18.94 meses $(D P=20.23)$, variando num intervalo entre 1 e 84 meses (i.e., 7 anos).

\section{Características do stalking pós-rutura}

Questionadas sobre se à data da realização do estudo continuavam a ser alvo de stalking por parte do ex-parceiro, a maioria das participantes respondeu positivamente: $32 \%(n=33)$ afirmou ter a "certeza que sim" e $20.4 \%(n=21)$ "pensava que sim". Por sua vez, $15.5 \%(n=16)$ tinha a "certeza que não" e $19.4 \%(n=20)$ "pensava que não". As restantes $(12.6 \%, n=$ 13) não sabiam se este tipo de vitimação teria ou não terminado.

No que concerne à duração do stalking, $10.6 \%(n=11)$ das participantes relatou durações inferiores a um mês, $28.8 \%(n=30)$ entre um a seis meses, $25 \%(n=26)$ entre sete a 12 meses, $22.1 \%(n=23)$ entre 13 meses a dois anos e $13.5 \%(n=14)$ referiu uma duração superior a dois anos.

Em média, as vítimas foram alvo de mais de 18 comportamentos de stalking diferentes $(M=18.75 ; D P=8.24)$, experienciados com uma elevada frequência $(M=1.65, D P=0.84)$. Na tabela 2 ilustra-se a percentagem relativa de cada um dos comportamentos e a frequência média das respetivas categorias. Para este efeito, recodificámos a frequência de cada um dos 35 itens que compõem o ICS-2 numa escala dicotómica ("Nunca" vs "Pelo menos uma vez") e ordenámo-los em função da sua tipologia. 
Tabela 2: Comportamentos de stalking sofridos após a rutura

$\%$ relativa $(N)$

Cortejamento \& Aproximação

Telefonou sem que lhe fosse pedido

$89.4(93)$

Procurou obter informações pessoais através dos meus amigos,

$85.6(89)$

familiares, colegas

Enviou ou deixou-me mensagens

$80.8(84)$

Enviou "toques" ou telefonou e desligou sem falar

$76.0(79)$

Abordou-me directamente

$72.1(75)$

Contactou e/ou tornou-se próximo dos meus amigos, familiares,

$63.5(66)$

colegas

Forçou encontros

$56.7(59)$

Expressou o seu afeto indirectamente

$54.8(57)$

Enviou, ou deixou para serem encontrados, cartas ou bilhetes

$37.5(39)$

Deu, ou deixou para serem encontrados, presentes

$28.8(30)$

Fez dedicatórias públicas

Frequência Média $(D P)$

$1.94(0.91)$

Assédio \& Invasão

Apareceu em locais ou nas proximidades de locais frequentados

$74.0(77)$

por mim

Vigiou ou controlou o meu comportamento

$70.2(73)$

Pediu a alguém para me vigiar ou obter informações sobre mim

$68.3(71)$

Divulgou informação prejudicial, embaraçosa ou falsa

$64.4(67)$

Perseguiu-me

$63.5(66)$

Forçou a entrada em minha casa, carro ou outro local onde eu

$51.9(54)$

estivesse

Leu ou roubou-me correspondência ou documentos pessoais

$51.9(54)$

Vasculhou a minha mochila, carteira, cacifo, lixo, etc.

$50.0(52)$

Roubou / apoderou-se de objetos pessoais

$50.0(52)$

Filmou ou tirou-me fotografias sem autorização

$15.4(16)$

Frequência Média (DP)

$1.75(1.02)$ 


\section{Ameaças \& Violência}

Maltratou-me verbalmente

Ameaçou-me verbalmente sobre o que me "poderia acontecer"

ou "poderia fazer"

Ameaçou fazer mal a si mesmo

Ameaçou ou assustou pessoas próximas de mim

Agrediu-me ou magoou-me fisicamente

Agrediu ou prejudicou pessoas próximas

Agarrou-me ou impediu-me de continuar o meu percurso

Manteve-me num local contra a minha vontade

Partiu, danificou ou destruiu objetos pessoais ou propriedades

Ameaçou-me com uma arma ou outro objeto intimidador

Forçou contacto sexual contra a minha vontade

Pediu a outras pessoas para me assustar, perseguir, ameaçar ou

maltratar

Atentou contra a minha vida

Maltratou ou matou os meus animais de estimação

A maioria das vítimas $(61.6 \%, n=61)$ avaliou a conduta de stalking como "um crime" e $33.3 \%(n=33)$ como "algo muito grave". Uma reduzida percentagem das inquiridas apresentou-se algo tolerante face à vitimação sofrida: $3 \%(n=3)$ considerou tratar-se de "algo que está errado mas que não é muito grave" e $2 \%(n=2)$ afirmou que é "algo normal".

Considerando a forma como as participantes avaliaram a vitimação, verificou-se existir uma correlação significativamente positiva entre o julgamento veiculado e a frequência média (global) dos comportamentos de stalking sofridos $\left(r_{s p}=.24, p=.019\right)$, ou seja, quanto mais as vítimas experienciaram comportamentos desta natureza, menos tolerantes se apresentaram face à conduta do ex-parceiro. Por sua vez, verificámos não existir uma relação significativa entre o julgamento veiculado e a duração do stalking $\left(r_{s p}=.03, p=.751\right)$.

Características da ex-relação íntima e do stalking pós-rutura: Que conexão?

Considerando a duração da ex-relação e a do stalking, verificou-se uma correlação significativamente positiva entre as variáveis $\left(r_{s p}=.26\right.$, 
$p=.008)$ : quanto mais duradoiro foi o relacionamento passado, mais prolongada foi a conduta de stalking pós-ruptura. Além disso, verificou-se existir também uma correlação significativamente positiva entre a duração da separação e a duração do stalking $\left(r_{s p}=.75, p<.001\right)$, sendo que separações mais prolongadas estavam associadas a casos de stalking mais longos.

De forma a obtermos uma compreensão clara acerca da relação entre as diferentes modalidades de vitimação, correlacionámos os comportamentos de stalking pós-rutura com a violência sofrida durante a relação entretanto cessada.

Tabela 3: Comportamentos violentos durante a ex-relação e comportamentos de stalking pós-rutura

Comportamentos de stalking pós-rutura

$\begin{array}{ccc}\text { Cortejamento \& } & \text { Assédio \& } & \text { Ameaças \& } \\ \text { Aproximação } & \text { Invasão } & \text { Violência } \\ \text { (Freq. Média }) & \text { (Freq. Média) } & \text { (Freq. Média) }\end{array}$

\begin{tabular}{l|llll} 
Maus-tratos & $\begin{array}{l}\text { V. Emocional } \\
\text { na ex-relação }\end{array}$ & $.08 \mathrm{~ns}$ & $.20^{*}$ & $.27^{* *}$ \\
(Freq. Média $)$ & & & \\
V. Física & $.04 \mathrm{~ns}$ & $.21^{*}$ & $.24^{*}$ \\
(Freq. Média $)$ & &
\end{tabular}

Nota. ${ }^{*} p<.05 ; * * p<.01$; ns: Não significativo $(p>.05)$; Os valores representam Coeficientes de Correlação de Spearman $\left(r_{s p}\right)$

Tal como se ilustra na Tabela 3, os comportamentos de stalking relativos a 'Assédio \& Invasão' e os relativos a 'Ameaças \& Violência' correlacionam-se significa e positivamente com a 'Violência Emocional' e com a 'Violência Física' sofridas durante a ex-relação. Especificamente, a correlação mais forte ocorreu entre estratégias de stalking relativas a 'Ameaças \& Violência' e a 'Violência Emocional', ao passo que a correlação mais fraca verificou-se entre os comportamentos de 'Assédio \& Invasão' e a 'Violência Emocional'. Por sua vez, os resultados demonstraram não existir associação significativa entre as estratégias de 'Cortejamento \& Aproximação' e qualquer um dos tipos de abuso experienciados na constância do relacionamento passado. 


\section{Respostas emocionais e coping face ao stalking pós-rutura}

$\mathrm{Na}$ sequência dos comportamentos de stalking, a maioria das vítimas $(67 \%, n=67)$ relatou sentir-se "muito assustada ou amedrontada". Menos de $1 / 4(24 \%, n=24)$ sentiu-se "um pouco assustada ou amedrontada" e 9\% ( $n=$ 9) sentiu-se "nada assustada ou amedrontada". Além disso, quase todas as participantes $(92.1 \%, n=93)$ relataram que a conduta do ex-parceiro exerceu uma influência negativa nas suas vidas: especificamente, $17.8 \%(n=18)$ afirmou que o stalking pós-rutura as afetou "um pouco", $26.7 \%(n=27)$ afirmou que afetou "muito" e 47.5\% $(n=48)$ afirmou que afetou "significativamente" as suas vidas.

Questionadas sobre o tipo de coping adotado face ao stalking, a esmagadora maioria das vítimas $(94.2 \%, n=98)$ referiu ter empreendido alguma estratégia. Destas, mais de metade afirmou ter procurado ajuda junto de amigos ou familiares $(66.3 \%, n=67)$, junto das autoridades policiais ou judiciais $(64.4 \%, n=65)$ e/ou optou por evitar o ex-parceiro $(55.4 \%, n=56)$. Menos de metade da amostra relatou outro tipo de estratégias, nomeadamente a negociação com o ex-parceiro, pedindo-lhe para que interrompesse aqueles comportamentos $(45.5 \%, n=46)$; a confrontação, assumindo uma postura intimidatória com aquele $(40.6 \%, n=41)$; e/ou a negação / minimização dos comportamentos sofridos (através do recurso à meditação, medicação, consumo de álcool ou outras substâncias para "esquecer") $(13.9 \%, n$ $=14)$. Em média, as vítimas implementaram entre 2 e 3 diferentes tipos de estratégias de coping $(M=2.96, D P=1.36)$.

Atendendo às sugestões apontadas na literatura acerca da potencial (in)adequação de diferentes tipos de estratégias (Spitzberg \& Cupach, 2001, 2007; Spitzberg, 2002), concluímos que 7.9\% $(n=8)$ das vítimas adotou unicamente estratégias consideradas inadequadas (negociar, confrontar e/ou negar/minimizar), $30.7 \%(n=31)$ adotou exclusivamente estratégias tidas como adequadas (evitar, procurar apoio junto de amigos / familiares e/ou junto das autoridades policiais / judiciais) e a maioria $(58.4 \%, n=59)$ implementou um conjunto de estratégias mistas (pelo menos, um tipo de estratégia considerada adequada e um tipo considerado inadequado).

\section{Impacto psicossocial e fatores preditores de desajustamento clínico}

No OQ-45.2, as participantes apresentaram um resultado total médio de $63.90(D P=24.69)$, variando num intervalo entre 15 e 134. Assim, e tomando como referência os valores normativos descritos por Machado \& Fassnacht (em preparação), $57.7 \%(n=60)$ das vítimas exibiu uma condição de ajustamento clínico e as restantes $(42.3 \%, n=44)$ manifestavam desajustamento clinicamente significativo. 
Impacto psicossocial e características sócio-demográficas

Os resultados demonstraram não existir diferenças significativas entre vítimas clinicamente ajustadas e desajustadas ao nível da idade $(t(102)=$ $-0.62, p=.536$ ). Por sua vez, encontrámos uma associação significativa entre as habilitações literárias das inquiridas (até ao $3^{\circ}$ ciclo do Ensino Básico vs mais do que o $3^{\circ}$ ciclo do Ensino Básico) e a respetiva condição clínica $\left(X^{2}(1)=8.79, p=.003\right)$, sendo que a maioria daquelas que terminou o secundário/curso superior (68\%) reportou desajustamento psicossocial clinicamente significativo, ao passo que a maioria daquelas $(65.8 \%)$ com graus de instrução inferior apresentou-se clinicamente ajustada. No que concerne à relação entre o nível de (des)ajustamento e o estatuto sócio - económico (Baixo/Médio-Baixo vs Médio/Médio-Alto), concluímos pela inexistência de qualquer associação significativa entre as variáveis $\left(X^{2}(1)=0.17, p=.683\right)$.

Impacto psicossocial e características da ex-relação

Considerando as características da ex-relação, concluímos que não existiam diferenças significativas entre os grupos ao nível da duração do relacionamento $(t(102)=0.70, p=.488)$ e ao nível da frequência média (global) dos comportamentos violentos sofridos durante aquele período de tempo $(t(102)=-0.88, p=.379)$.

Impacto psicossocial e características do stalking pós-rutura

Por sua vez, atendendo às características do stalking, não foi encontrada qualquer associação significativa entre o curso da vitimação e o nível de impacto psicossocial exibido $\left(X^{2}(4)=3.34, p=503\right.$ ), assim como entre aquela variável de sintomatologia e a duração da experiência (menor que 6 meses $v s$ maior ou igual a 6 meses; $X^{2}(1)=2.20, p=.138$ ). Já em relação à frequência média (global) dos comportamentos de stalking, verificou-se uma diferença estatisticamente significativa entre os grupos em análise $(t(102)=$ $-3.13, p=.002)$ : as vítimas que exibiram desajustamento clínico relataram ter sido alvo de comportamentos desta natureza mais frequentemente $(M=$ 1.94; $D P=0.78)$ do que aquelas que não exibiram a mesma condição clínica $(M=1.44 ; D P=0.82)$.

Impacto psicossocial e tipo de estratégias de coping adotadas

Analisando a relação entre o tipo de coping e o nível de impacto exibido, e tal como se ilustra na Tabela 4, encontrou-se uma associação estatisticamente significativa entre a sintomatologia e a resposta de evitamento: a maioria das vítimas que evitou o ex-parceiro exibiu ajustamento clínico e a maioria daquelas que não adotou esta estratégia manifestou desajustamento com relevância clínica. Também foi encontrada uma associação estatistica- 
mente significativa entre a estratégia de confrontação e o nível de impacto psicossocial: a maioria das vítimas que confrontou o stalker apresentou desajustamento clínico, ao contrário da maioria daquelas que não empreendeu este tipo de estratégia.

Tabela 4: Nível de impacto e tipo de estratégias de coping adotadas

\begin{tabular}{|c|c|c|c|c|}
\hline & & $\begin{array}{l}\text { Vítimas clinicamen- } \\
\text { te AJUSTADAS } \\
(n=58)\end{array}$ & $\begin{array}{l}\text { Vítimas clinicamen- } \\
\text { te DESAJUSTADAS } \\
\quad(n=43)\end{array}$ & $X^{2}(1)$ \\
\hline \multirow[t]{2}{*}{$\begin{array}{l}\text { Ajuda Ami- } \\
\text { gos/Família }\end{array}$} & NÃO & $50 \%(17)$ & $50 \%(17)$ & \multirow{2}{*}{$1.16 \mathrm{~ns}$} \\
\hline & SIM & $61.2 \%(41)$ & $38.8 \%(26)$ & \\
\hline \multirow[t]{2}{*}{ Ajuda Polícia } & NÃO & $69.4 \%(25)$ & $30.6 \%(11)$ & \multirow[b]{2}{*}{$3.31 \mathrm{~ns}$} \\
\hline & SIM & $50.8 \%(33)$ & $49.2 \%(32)$ & \\
\hline \multirow[t]{2}{*}{ Evitar } & NÃO & $44.4 \%(20)$ & $55.6 \%(25)$ & \multirow[b]{2}{*}{$5.60 *$} \\
\hline & SIM & $67.9 \%(38)$ & $32.1 \%(18)$ & \\
\hline \multirow[t]{2}{*}{ Negociar } & NÃO & $65.5 \%(36)$ & $34.5 \%(19)$ & \multirow[b]{2}{*}{$3.18 \mathrm{~ns}$} \\
\hline & SIM & $47.8 \%(22)$ & $52.2 \%(24)$ & \\
\hline \multirow[t]{2}{*}{ Confrontar } & NÃO & $68.3 \%(41)$ & $31.7 \%(19)$ & \multirow[b]{2}{*}{$7.19^{* *}$} \\
\hline & SIM & $41.5 \%(17)$ & $58.5 \%(24)$ & \\
\hline \multirow[t]{2}{*}{ Negar/Minimizar } & NÃO & $57.5 \%(50)$ & $42.5 \%(37)$ & \multirow[b]{2}{*}{$0.00 \mathrm{~ns}$} \\
\hline & SIM & $57.1 \%(8)$ & $42.9 \%(6)$ & \\
\hline
\end{tabular}

Nota. ${ }^{*} p<.05 ; * * p<.01$, ns: Não significativo $(p>.05)$

Preditores de desajustamento psicossocial clinicamente significativo

A regressão logística (método Enter) foi usada para proceder à previsão do desajustamento clínico a partir das variáveis que, nas análises anteriores, se mostraram significativamente relacionadas com o nível de impacto psicossocial. Refira-se que as análises preliminares revelaram estarem cumpridos os pressupostos subjacentes à utilização deste tipo de análise, nomeadamente dimensão adequada da amostra, ausência de multicolinearidade e de outliers (Pallant, 2007). 
Os preditores foram inseridos em três blocos, tal como se ilustra na Tabela 5: no primeiro bloco foram inseridas as características sócio-demográficas das vítimas (i.e., habilitações literárias), no segundo introduziram-se as características da vitimação por stalking (i.e., frequência média global dos comportamentos) e no terceiro integraram-se as respostas de coping (i.e., evitar e confrontar).

Tabela 5: Preditores de desajustamento psicossocial clinicamente significativo

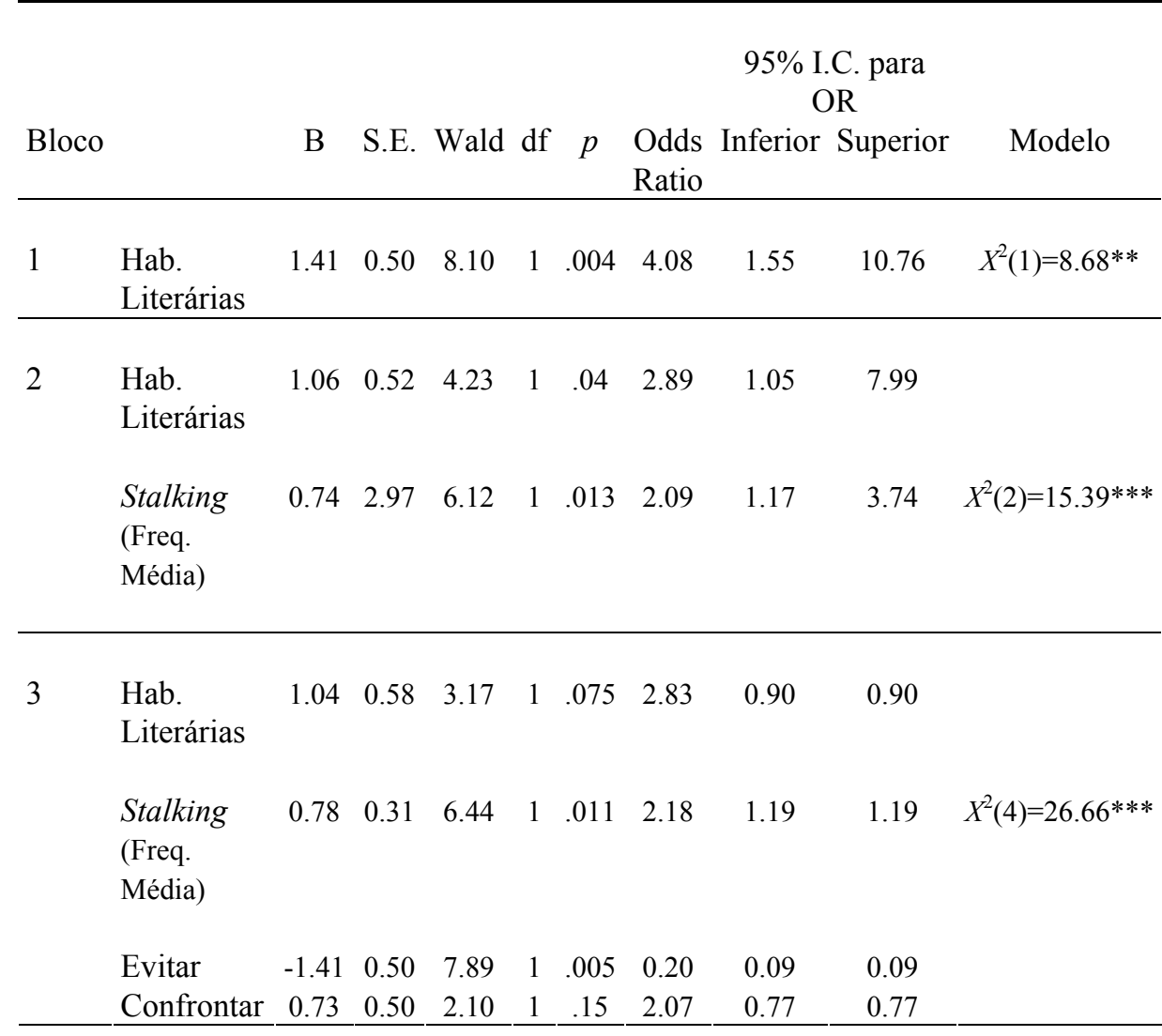

Nota. Variável a predizer: Desajustamento psicossocial clinicamente significativo $(0=$ Sem desajustamento, $1=$ Com desajustamento)

Preditores dicotómicos: Habilitações literárias $\left(0=\right.$ até ao $3^{\circ}$ ciclo do Ensino Básico, $1=$ mais do que o $3^{\circ}$ ciclo do Ensino Básico); Evitar ( $0=$ Não, $\left.1=\operatorname{Sim}\right)$; Confrontar $(0=\mathrm{Não}, 1=\mathrm{Sim})$

$* * \mathrm{p}<.01 ; * * * \mathrm{p}<.001 ;$ ns: Não significativo $(p>.05)$ 
O modelo com as características da vítima (i.e., habilitações literárias) explicou entre $8.5 \%$ e $11.4 \%$ da variância (Cox \& Snell $R$ Square e Nagelkerke $R$ Square, respetivamente) e classificou corretamente $66.3 \%$ dos casos. As habilitações literárias da vítima assumiram uma contribuição individual estatisticamente significativa na predição do desajustamento psicossocial, sendo que as inquiridas com graus de formação mais elevados evidenciaram maior probabilidade de exibir aquela condição clínica.

A introdução da frequência média dos comportamentos de stalking sofridos contribuiu para aumentar ligeiramente o poder preditivo do modelo, o qual passou a explicar entre $14.5 \%$ e $19.5 \%$ da variância (Cox \& Snell $R$ Square e Nagelkerke R Square, respetivamente). Ainda assim, o número de casos corretamente classificados sofreu um ligeiro decréscimo (64.5\%). Ambas as variáveis revelaram-se preditores significativos do desajustamento psicossocial: mais uma vez, as vítimas com habilitações mais elevadas evidenciaram maior probabilidade de exibir aquela condição clínica, o mesmo acontecendo com aquelas que sofreram comportamentos de stalking mais frequentemente.

As variáveis incluídas no terceiro bloco, relativas às estratégias de coping adotadas face a esta experiência de vitimação, vieram acrescentar um contributo ainda mais importante na predição do desajustamento psicossocial, tendo o modelo passado a explicar de $23.8 \%$ a $32 \%$ da variância (Cox \& Snell $R$ Square e Nagelkerke $R$ Square, respetivamente) e a classificar corretamente $69.4 \%$ dos casos. Neste caso, apenas dois dos preditores incluídos exerceram uma contribuição individual estatisticamente significativa na predição daquela condição: a frequência média dos comportamentos de stalking (preditor mais forte) e a estratégia de evitamento. Pudemos concluir que as vítimas que foram mais frequentemente alvo daquele tipo de comportamentos e aquelas que não evitaram os contactos com o stalker tinham maior probabilidade de manifestar desajustamento psicossocial com relevância clínica.

\section{Discussão}

Assumindo particular relevância dada a escassez de investigação em Portugal sobre esta temática, os resultados deste estudo revelam uma realidade preocupante sobre as mulheres vítimas de violência doméstica e de stalking por parte do ex-parceiro.

A análise da vitimação por stalking após a separação ilustra conceções amplamente documentadas na literatura sobre o tema, entre as quais se destaca a sua natureza altamente prolongada: mais de $60 \%$ da amostra foi alvo deste tipo de violência durante 6 ou mais meses, tendo $13.5 \%$ das inquiridas reportado uma duração superior a 2 anos. Esta evidência está de acordo com 
a representação traduzida nos diferentes estudos internacionais conduzidos junto desta população (e.g., Brewster, 1998; Mechanic, Weaver, \& Resick, 2002) e suporta a ideia de que, neste contexto em particular, o risco de uma vitimação persistente e continuada é expressivo (cf. McEwan et al., 2007). No que concerne à natureza dos comportamentos, os resultados são também bastante esclarecedores e concordantes com a literatura internacional (Spitzberg \& Cupach, 2007), sendo possível identificar um continuum comportamental que abrange ações com uma gravidade bastante distinta entre si. Especificamente, os comportamentos mais comuns (reportados por mais de $70 \%$ das participantes), incluíram telefonemas ou outro tipo contacto telefónico indesejado, tentativas para obter informações pessoais sobre a vítima através de terceiros, abordagens diretas, aparecimento nos locais ou nas proximidades dos locais frequentados por aquela, vigilância ou controlo do seu comportamento, assim como agressões e ameaças, ambas de natureza verbal. Importa sublinhar que o caráter coberto de muitas destas ações poderá colocar os seus intervenientes em posições paradoxais: se, para o stalker, os riscos envolvidos são menores (e.g., ausência de tipificação criminal para vários destes atos), para a vítima torna-se mais difícil proteger-se das mesmas (e.g., maior dificuldade de ver as suas necessidades acreditadas por terceiros). Para além disso, estes resultados sugerem que o stalking tem na sua génese uma forma de violência mais camuflada ou "invisível", hipótese que vai ao encontro das indicações também apontadas por outros autores (e.g., Mechanic, Weaver, \& Resick, 2002).

Ainda assim, os comportamentos sofridos durante a campanha de stalking pós-rutura situados no pólo mais extremo do continuum comportamental foram muito elevados: atos de violência física $(53.8 \%)$, ameaças com armas ou outros objetos intimidatórios $(37.5 \%)$ ou ações contra a sua própria vida $(29.8 \%)$. Estes resultados corroboram evidências documentadas noutros trabalhos, nomeadamente aqueles que identificam a presença de ameaças (no geral) como um dos preditores mais significativos para a ocorrência de abuso físico (e.g., Brewster, 2002; Roberts, 2005) e aqueles que apresentam o cenário de stalking no âmbito de relações íntimas como o de maior risco (cf., McEwan et al., 2007). De realçar ainda o facto de, não raras vezes, este tipo de ofensores ter envolvido terceiros na campanha de assédio perpetrada, quer como seus aliados, quer como alvos secundários dos seus comportamentos, $\mathrm{o}$ que é igualmente compatível com os dados disponíveis na literatura (e.g., Melton, 2007).

A esmagadora maioria das vítimas avaliou a conduta de stalking sofrida como "um crime" ou "algo muito grave". Os julgamentos menos tolerantes estavam associados a experiências caracterizadas por uma maior reiteração deste tipo de comportamentos. Julgamos que outros fatores podem ter contribuído para esta atitude geral de reprovação da campanha de assédio 
pós-rutura, nomeadamente o acompanhamento institucional recebido e, sobretudo, a história passada de violência perpetrada pelo mesmo agente. Ao violar as expectativas das mulheres face à reserva, edificação e preservação do "seu próprio território" (Wuest \& Merrit-Gray, 1999, p. 118), este tipo de conduta poderá ser vista como um obstáculo à construção de um projeto de vida alternativo e imune à violência, pondo em causa os propósitos que, possivelmente, presidiram à decisão de separação.

As conclusões sobre a relação entre diferentes comportamentos abusivos ocorridos durante a ex-relação e diferentes comportamentos de stalking ocorridos após a rutura constituem mais um alicerce para a conceptualização do stalking como uma faceta da violência na intimidade (e.g., Basile \& Hall, 2010; Miller, 2006). A este respeito, não podemos ignorar a aproximação conceptual entre os comportamentos de 'Cortejamento \& Aproximação' e as estratégias que, tipicamente, caracterizam a fase de "lua-de-mel" do ciclo da violência (Walker, 1994): durante a relação, este tipo de atos serve para o ofensor envolver a vítima de "bons tratos", investindo na sua sedução através de estratégias de manipulação; contudo, durante aquele período de tempo, a mulher vítima raramente tem consciência do nível de manipulação envolvido naquele tipo de atos e, em virtude disso, não lhes reconhece um caráter abusivo. Contrariamente, porque a separação traduz o seu desejo em cessar os contactos com o ex-parceiro, aqueles atos de cortejamento e sedução passam agora a não ser bem recebidos pela mulher e esta parece estar assim mais capaz de os perceber como intrusivos e manipulativos.

A forma como as vítimas respondem à experiência de stalking constitui outra dimensão importante deste estudo. Desde logo, importa sublinhar que quase todas as participantes $(91 \%)$ relataram algum nível de medo na sequência dos comportamentos perpetrados pelo ex-parceiro, levando-nos a concluir pelo caráter endémico desta reação, sendo estes dados corroborados pela investigação internacional (e.g., Brewster, 1998). Além disso, a esmagadora maioria das participantes $(92.1 \%)$ reconheceu que este tipo de vitimação pós-rutura teve uma influência negativa nas suas vidas, reforçando o efeito nocivo destas experiências (e.g., Mechanic, 2002).

Ainda assim, as vítimas demonstraram ser ativas na gestão individual da violência sofrida, empreendendo diferentes tipos de estratégias de coping face aos comportamentos de stalking perpetrados pelo ex-parceiro. Embora tenhamos percebido que a maioria acabou por adotar, simultaneamente, estratégias tidas como positivas e outras menos eficazes (Spitzberg \& Cupach, 2001, 2007; Spitzberg, 2002), as respostas mais frequentemente apontadas pelas participantes correspondem aos estilos de coping recomendados pela literatura, situação que pode ser compreendida pelo acompanhamento institucional de que todas usufruíram. Além disso, o facto de acumu- 
larem a vitimação doméstica anterior pode explicar também o seu maior sucesso e expertise na gestão da violência pós separação.

Em termos psicossociais, $57.7 \%$ das vítimas encontrava-se clinicamente ajustada, contrariamente às restantes $42.3 \%$, onde foi identificado desajustamento com relevância clínica. A condição sub-clínica manifestada por mais de metade da amostra pode ser compreendida de diferentes formas, entre as quais o pressuposto de "anestesia ao mal-estar" (Ravazola, 1997). Julgamos, contudo, que esta explicação é pouco compatível com a reduzida tolerância face à conduta de stalking evidenciada pela esmagadora maioria das participantes. Provavelmente, estas mulheres possuem fatores de resiliência pessoal e/ou dispõe de outras circunstâncias protetoras (e.g., apoio e suporte informal mais efetivo, autoconceito positivo) que lhes permitiram preservar ou recuperar a sua condição psicossocial, apesar das dificuldades e adversidades enfrentadas (Spitzberg, 2002). Além disso, importa também atender ao tempo de separação e à possibilidade de reorganização das suas vidas graças ao apoio institucional recebido.

Por fim, de modo a identificarmos os preditores de desajustamento clínico, conduzimos uma análise de regressão logística, com base nas variáveis que relevaram uma relação estatisticamente significativa com aquela variável de sintomatologia em análises prévias.

Assim, quando se analisaram exclusivamente as características da vítima (i.e., habilitações literárias), concluímos que as vítimas com um grau de formação mais elevado (ensino secundário e/ou universitário) tinham maior probabilidade de exibir desajustamento psicossocial clinicamente significativo. Este dado poderá ser explicado pelo facto da maior instrução facilitar a perceção do caráter intrusivo e violador das liberdades individuais que os comportamentos de stalking encerram.

Quando se exploraram de forma conjunta as características da vítima e as dinâmicas do stalking (i.e., frequência média global dos comportamentos), constatámos que ambas as variáveis eram preditores do desajustamento psicossocial, sendo que as vítimas que sofreram comportamentos de stalking mais frequentemente apresentaram maior probabilidade de manifestar aquela condição clínica. Estes resultados vão ao encontro das conclusões de outros trabalhos (e.g., Mechanic, Uhlmansiek et al., 2002) e demonstram que esta modalidade de vitimação assume um papel particularmente crítico na explicação da sintomatologia exibida por mulheres alvo de múltiplas experiências de violência no contexto relacional, especialmente quando os comportamentos de stalking assumem um formato altamente reiterado, situação que pode ser explicada pelo seu caráter mais imprevisível e pela prolongada exposição à ameaça que impõe (e.g., Collins \& Wilkas, 2001).

A análise conjunta das características da vítima, das dinâmicas de stalking e do tipo de estratégias de coping adotadas pelas participantes (i.e., 
evitar e confrontar) permitiu concluir que o desajustamento psicossocial exibido por vítimas de stalking pós-rutura resulta de uma interação complexa de variáveis de naturezas várias. Neste último caso, apenas dois dos preditores incluídos exerceram uma contribuição individual estatisticamente significativa na predição: a frequência média dos comportamentos de stalking (preditor mais forte) e a estratégia de evitamento. Em relação a este último, sucedeu que as vítimas que não evitaram os contactos com o stalker tinham maior probabilidade de manifestar desajustamento psicossocial com relevância clínica. Este resultado sugere que o tipo de coping utilizado pode exercer um importante papel mediador na relação entre a vitimação por stalking e o desajustamento psicossocial exibido pelas vítimas e fornece sustentação empírica às conceções que apresentam esta estratégia como uma das mais eficazes em situações de stalking (Spitzberg \& Cupach, 2001, 2007; Spitzberg, 2002). O evitamento dos contactos, não só dificulta a tarefa ao stalker e diminui o risco de revitimação, como pode favorecer sentimentos de autoeficácia na gestão desta experiência.

Neste sentido, os resultados deste trabalho sugerem que a intervenção com vítimas de stalking por parte de ex-parceiros deve contemplar um conjunto específico de saberes, competências e conhecimentos. Intervir "às cegas" ou importando práticas de outros contextos, não só é pouco eficaz como encerra possíveis incorreções, dadas as idiossincrasias desta modalidade de vitimação. A este nível, destaca-se a necessidade de o profissional desenvolver junto da vítima estratégias adequadas para lidar com o stalker. Especificamente, importa reforçar junto daquela a necessidade e pertinência de transmitir ao stalker o seu desinteresse e desagrado face à atenção recebida, através de uma mensagem breve, explícita, não emotiva e desprovida de justificações; depois de transmitido o desinteresse, a vítima deverá ser sensibilizada para a importância de cessar todos os contactos com o stalker, de modo a não reforçar os seus comportamentos, nem contribuir inadvertidamente para a sua perpetuação (Matos, Grangeia, Ferreira, \& Azevedo, 2011).

Este estudo permitiu corroborar evidências internacionais já disponíveis sobre o stalking no contexto de relações íntimas, chamando a atenção para uma realidade que, embora seja comummente relatada pelas mulheres vítimas de maus tratos conjugais, continua votada a alguma negligência no panorama nacional. Interessa, pois, aperfeiçoar o conhecimento nacional desta realidade e investir na melhoria das políticas de intervenção, capazes de patrocinar respostas efetivas às múltiplas necessidades enfrentadas pelas vítimas de violência no contexto de relacionamentos íntimos.

Não obstante os contributos alcançados, importa refletir sobre algumas limitações deste trabalho. $\mathrm{O}$ facto de termos utilizado uma amostra de conveniência inviabiliza, desde logo, a generalização dos resultados obtidos. Outra limitação deste estudo prende-se com a particular ênfase conferida aos 
atos de violência emocional e física ocorridos durante a ex-relação, deixando por abordar a violência sexual durante aquele período de tempo; esta limitação é inerente à medida utilizada (IVC-3), não deixando de ter sido ponderada aquando da sua escolha (que, ainda assim, foi selecionada dado tratar-se de um instrumento validado e aferido para a população portuguesa). Além disso, o facto de a amostra ter sido recolhida em instituições formais poderá ter potenciado o contacto com casos mais extremos, caracterizados precisamente pela experiência cumulativa de diferentes modalidades de vitimação e por níveis elevados de dificuldades psicossociais. Finalmente, outra reserva deste estudo prende-se com o seu design retrospetivo.

\section{Referências}

Basile, K. C. \& Hall, J. (2010). Intimate partner violence perpetration by court-ordered men: Distinctions and intersections among physical violence, sexual violence, psychological abuse and stalking. Journal of Interpersonal Violence. DOI: $10.1177 / 0886260510362896$.

Brewster, M. P. (1998). An exploration of the experiences and needs of former intimate stalking victims. Final report submitted to the National Institute of Justice. West Chester, PA: Wester Chester University.

Brewster, M. P. (2002). Stalking by former intimates: Verbal threats and other predictors of physical violence. In K. E. Davies, I. H. Frieze, \& R. D. Maiuro (Eds.), Stalking. Perspetives on Victims and Perpetrators (pp. 292-311). New York: Springer Publishing Company.

Burgess, A. W., Harner, H., Baker, T., Hartman, C. R., \& Lole, C. (2001). Batterers stalking patterns. Journal of Family Violence, 16(3), 309-321.

Campbell, J. C., Glass, N., Sharps, P. W., Laughon, K., \& Bloom, T. (2007). Intimate partner homicide. Review and implications of research and policy. Trauma, Violence, \& Abuse, 8(3), 246-269.

Collins, M. J. \& Wilkas, M. B. (2001). Stalking trauma syndrome and the traumatized victim. In J. A. Davies (Ed.), Stalking crimes and victim protection. Prevention, intervention, threat assessment, and case management (pp. 317-334). Florida: CRC Press.

Dutton, L. B. \& Winstead, B. A. (2010). Types, frequency, and effectiveness of responses to Unwanted Pursuit and Stalking. Journal of Interpersonal Violence, 20(10), 1-28.

Fife-Schaw, C. (2000). Levels of Measurement. In G. M. Breakwell, S. Hammond, \& C. Fife-Schaw (Eds.), Research Methods in Psychology (2rd Edition, pp. 147-157). London: Sage.

Grangeia, H. \& Matos, M. (em preparação). How far does love go? Gendered perspectives on unwanted relational pursuit. 
Grangeia, H. \& Matos, M. (2010). Stalking: Consensos e Controvérsias. In C. Machado (Coord.), Novos olhares sobre a vitimação criminal: teorias, impacto e intervenção (pp. 121-166). Braga: Psiquilíbrios.

Grangeia, H., Matos, M., \& Machado, C. (2008). Inventário de Comportamentos de Stalking. In C. Machado, L. Almeida, M. Gonçalves, V. Ramalho, \& S. Martins (Eds.), XII Atas do Congresso Internacional Avaliação Psicológica: Formas e Contextos. Braga: Psiquilíbrios Edições.

Kamphuis, J. H. \& Emmelkamp, P. M. G., \& Bartak, A. (2003). Individual differences in post-traumatic stress following post-intimate stalking: Stalking severity and psychosocial variables. British Journal of Clinical Psychology, 42, 145$-156$.

Logan, TK \& Walker, R. (2009). Partner stalking. Psychological dominance or "business as usual"?. Trauma, Violence, \& Abuse, 10(3), 247-270.

Logan, TK, Shannon, L., Cole, J., \& Walker, R. (2006). The impact of differential patterns of physical violence and stalking on mental health and help-seeking among women with protective orders. Violence Against Women, 12(9), 866-886 .

Lowney, K. S. \& Best, J. (1995). Stalking strangers and lovers: changing media typifications of a new crime problem. In J. Best (Ed.), Images of issues: Typifying contemporary social problems (pp. 33-57). New York: Aldine de Gruyter.

Machado, C., Matos, M. \& Gonçalves, M.M. (2006). Inventário de Violência Conjugal. In Machado, C., Matos, M. \& Gonçalves, M.M. (Eds.). Manual da Escala de Crenças sobre Violência Conjugal e do Inventário de Violência Conjugal (pp. 12-14). Braga: Psiquilíbrios Edições.

Matos, M., Grangeia, H., Ferreira, C., \& Azevedo, V. (2011). Stalking: Boas práticas no apoio à vítima. Manual para profissionais. Lisboa: Comissão para a Cidadania e Igualdade de Género.

McEwan, T. E., Mullen, P. E., \& Purcell, R. (2007). Identifying risk factors in stalking: review of current research. International Journal of Law and Psychiatry, 30, 1-9.

Mechanic, M. B. (2002). Stalking victimization: Clinical implications for assessment and intervention. In K. E. Davis, I. H. Frieze, \& R. D. Maiuro (Eds.), Stalking: Perspetives on victims and perpetrators (pp. 31-61). New York: Springer Publishing Company.

Mechanic, M. B., Uhlmansiek, M. H., Weaver, T. L., \& Resick, P. A. (2002a). The impact of severe stalking experienced by acutely battered women: An examination of violence, psychological symptoms and strategic responding. In K. E. Davis, I. H. Frieze, \& R. D. Maiuro (Eds.), Stalking: Perspetives on victims and perpetrators (pp. 89-111). New York: Springer Publishing Company.

Mechanic, M. B., Weaver, T. L., \& Resick, P. A. (2002b). Intimate partner violence and stalking: Exploration of patterns and correlates in a sample of acutely battered women. In K. E. Davis, I. H. Frieze, \& R. D. Maiuro (Eds.), Stalking: Perspetives on victims and perpetrators (pp. 62-88). New York: Springer Publishing Company.

Meloy, J. R. (2007). Stalking: The state of the science. Criminal Behaviour and Mental Health, 17, 1-7. 
Melton, H. C. (2007). Stalking in the context of intimate partner abuse. In the victims' words. Feminist Criminology, 2(4), 347-363.

Miller, J. A. (2006). A specification of the types of intimate partner violence experienced by women in the general population. Violence Against Women, 12(12), 1105-1131.

Mohandie, K., Meloy, J. R., McGowan, M. G., \& Williams, J. (2006). The RECON typology of stalking: Reliability and validity based upon a large sample of North American stalkers. Journal of Forensic Sciences, 51, 147-155.

Morewitz, S. J. (2003). Stalking and violence: New patterns of trauma and obsession. New York: Kluwer Academic / Plenum Publishers.

Mullen, P. E., Pathé, M., \& Purcell, R. (2001). Stalking: New constructions of human behaviour. Australian and New Zealand of Psychiatry, 35, 9-16.

O’Connor, M. \& Rosenfeld, B. (2004). Introduction to the special issue on stalking. Finding and filling the empirical gaps. Criminal Justice and Behavior, 31(1), 3-8 .

Machado, P. P. P. \& Fassnacht, D. (em preparação). The Outcome Questionnaire (OQ-45) in a Portuguese Population: Psychometric Properties, ANOVAS, and Confimatory Factory Analysis.

Pallant, J. (2007). SPSS. Survival Manual. A step by step guide to date analysis using SPSS for Windows (3th Edition). London: McGraw-Hill.

Ravazola, M. C. (1997). Historias infames: Los maltratos en las relaciones. Buenos Aires: Paidós Terapia Familiar.

Roberts, K. A. (2005). Women's experience of violence during stalking by former romantic partners. Violence Against Women, 11(1), 89-114.

Sheridan, L. P., Blaauw, E., \& Davies, G. M. (2003). Stalking: knowns and unknowns. Trauma, Violence, \& Abuse, 4, 148-162.

Spitzberg, B. H. \& Cupach, W. R. (2001). Paradoxes of pursuit: Toward a relational model of stalking-related phenomena. In J. A. Davies (Ed.), Stalking crimes and victim protection. Prevention, intervention, threat assessment, and case management (pp. 97-136). Florida: CRC Press.

Spitzberg, B. H. \& Cupach, W. R. (2007). The state of art of stalking: Taking stock of the emerging literature. Aggression and Violent Behavior, 12, 64-86.

Spitzberg, B. H. (2002). The tactical topography of stalking victimization and management. Trauma, Violence, \& Abuse, 3, 261-288.

Walker, L. E. A. (1994). Abused Women and Survivor Therapy: A practical guide for the psychotherapist. Washington D. C.: American Psychological Association.

Wuest, J. \& Merrit-Gray, M. (1999). Not going back: Sustaining the separation in the process of leaving abusive relationships. Violence Against Women, 5(2), $110-133$. 